\title{
Subject-specific thumb muscle activity during functional tasks of daily life
}

\author{
Faes D. Kerkhof*, Gertjan Deleu, Priscilla D’Agostino, Evie E. Vereecke \\ Jan Palfijn Anatomy Lab, Department of Development and Regeneration, KU Leuven, Belgium
}

\section{A R T I C L E I N F O}

\section{Article history:}

Received 5 January 2016

Received in revised form 15 June 2016

Accepted 18 June 2016

\section{Keywords:}

Trapeziometacarpal joint

EMG

Fine-wire

Intramuscular

Osteoarthritis

\begin{abstract}
A B S T R A C T
Background: The trapeziometacarpal joint is subjected to high compressive forces during powerful pinch and grasp tasks due to muscle loading. In addition, muscle contraction is important for stability of the joint. The aim of the present study is to explore if different muscle activation patterns can be found between three functional tasks.

Methods: Isometric forces and fine-wire electromyographic (fEMG) activity produced by three intrinsic and four extrinsic thumb muscles were measured in 10 healthy female volunteers. The participants performed isometric contractions in a lateral key pinch, a power grasp and a jar twist task. The tasks were executed with and without EMG recording to verify if electrode placement influenced force production. Results: A subject-specific muscle recruitment was found which remained largely unchanged across tasks. Extrinsic thumb muscles were significantly more active than intrinsic muscles in all tasks. Insertion of the fEMG electrodes decreased force production significantly in all tasks.

Conclusion: The thumb muscles display a high variability in muscle activity during functional tasks of daily life. The results of this study suggest that to produce a substantial amount of force, a wellintegrated, but subject-specific, co-contraction between the intrinsic and extrinsic thumb muscles is necessary.
\end{abstract}

(c) 2016 Elsevier Ltd. All rights reserved.

\section{Introduction}

The human thumb plays a key role in normal hand function. The trapeziometacarpal (TMC) joint is the osteoarticular basis from which the opposable thumb is able to perform its numerous functions and movements. The specific geometry of this joint allows multiplanar movement (Eaton et al., 2000; Edmunds, 2011). While the TMC joint is loose and unstable during a resting posture, the joint is subjected to high compressive forces during powerful pinch and grasp. Cooney and Chao (1977) estimated joint forces of 88$127 \mathrm{~N}$ during lateral key pinch and 834-1608 $\mathrm{N}$ during power grasp which, combined with the small articular surface of the TMC joint (ca. $130 \mathrm{~mm}^{2}$ ), lead to elevated contact pressures. High mechanical loading at the joint can lead to the development of osteoarthritis (OA). In contrast to weight-bearing joints, such as the knee joint, the mechanical loading of the TMC joint is largely generated by contraction of intrinsic and extrinsic thumb muscles.

\footnotetext{
* Corresponding author at: Jan Palfijn Anatomy Lab, Department of Development and Regeneration, KU Leuven, Etienne Sabbelaan 53, 8500 Kortrijk, Belgium.

E-mail address: Faes.Kerkhof@kuleuven.be (F.D. Kerkhof).
}

It is, therefore, of high clinical relevance to investigate thumb muscle activations during functional tasks.

Muscle activity and muscle activation patterns are typically investigated using surface electromyography (sEMG) or fine-wire electromyography (fEMG) (Konrad, 2005; Türker, 1993). Finewire EMG seems to be the most appropriate method to document the function of muscle groups which have a deep location and small size, such as the intrinsic thumb muscles (Corneil et al., 2012; Rudroff et al., 2008). In addition, cross-talk is limited as fEMG-electrodes are implanted directly into the muscle, contrary to sEMG where the electrodes are applied to the overlying skin (Solomonow et al., 1994). Even though several studies used fEMG to investigate the muscle activity of the thumb muscles (Burgar et al., 1997; Johanson et al., 2001; Valero-Cuevas et al., 1998), muscle activation patterns for specific functional tasks remains inconclusive.

To get a better insight into the role of the thumb muscles during activities of daily living and their relation with the development of $\mathrm{OA}$ at the TMC joint, baseline muscle activation patterns in healthy persons need to be established. The aim of this study is to evaluate if specific muscle activation patterns can be recognized during a lateral key pinch, a power grasp and a jar twist task, by analysing 
the activity of the relevant intrinsic or extrinsic muscles. Based on the muscle function (Cooney et al., 1985; Maier and HeppReymond, 1995), we hypothesize that there will be a taskspecific activation of the thenar muscles, with strong activation of the thumb adductors and flexors during lateral key pinch and co-contraction of the pollical adductors and abductors during power grasp and jar twist. Furthermore, we expect to see a higher recruitment of the intrinsic thenar muscles during lateral key pinch and a relatively higher activity of the extrinsic thenar muscles during grip tasks such as power grip and jar twist. Finally, we expect to see co-contraction of the antagonistic muscles during all tasks to stabilize the TMC joint.

\section{Materials and methods}

\subsection{Participants}

Eleven healthy young women (mean age: 22.88 years \pm SD: 4.75 years) volunteered for this study. Volunteers with musculoskeletal problems in the forearm or hand region, previous trauma or surgery in this area or neurological conditions were excluded from the study. Approval from the medical ethical commission of the University of Leuven was obtained prior to the start of the project (ML9602) and an informed consent was signed by each participant.

\subsection{Force data collection}

Force data were collected using a custom-made polycarbonate jig (Orthopaedic Bioengineering Laboratories, Brown University/ Rhode Island Hospital, Providence, RI, USA) with an embedded load cell (0-50 lb, Model D, Thrue-Hole, Honeywell International Inc., NJ, USA) (Fig. 1). Using a universal inline amplifier (model UV, Sensotec, Columbus, USA), force data were recorded using customwritten data acquisition software (Labview, Texas Instruments, Austin, USA). This experimental setup and protocol have been used extensively in other studies (Crisco et al., 2015; Halilaj et al., 2014, 2013).

The participants performed isometric contractions for $5 \mathrm{~s}$ with their dominant hand during three tasks; a lateral key pinch, a power grasp and a jar twist task. The exact positioning of the hand and thumb in each task is shown in Fig. 1A-C. Each task was executed on two different effort levels using visual feedback: two maximal isometric contractions and one contraction at $80 \%$ of maximal force (Submax). The tasks were executed before and after electrode placement to evaluate the influence of electrode insertion on force production.

\subsection{EMG data collection}

Electromyographic (EMG) data were collected using an 8channel Trigno wireless system (Delsys, Inc., Boston, USA) equipped with fine-wire electrodes. The raw EMG signal was recorded with an amplifier band pass of $2000 \mathrm{~Hz}$, an A/D conversion sampling rate of $4000 \mathrm{~Hz}$ and a gain setting between 1 and 300. The fine-wire electrodes consisted of pairs of disposable, bipolar, sterilized, $50 \mathrm{~mm}$ long, hooked wire electrodes with a 25 gauge needle as a cannula (Motion Lab Systems, Inc., LA, USA). The wire were made out of 304 series stainless steel with a green nylon insulation with $5 \mathrm{~mm}$ bare-wire terminations.

The skin of the participant's dominant forearm and hand was disinfected before insertion of the electrodes. An ultrasoundguided technique based on the recommendations of Perotto was used to insert the electrodes in each of the eight target muscles (Perotto, 1994). Ultrasound images were obtained with a S8Sonoscape portable digital color Doppler ultrasound system (Sonoscape, Shenzhen, China) using a linear, L-shaped hockey stick probe (probe 10l2, 6-11 Hz, $19 \mathrm{~mm}$ ). This procedure was executed by a hand surgeon (PDA). The ends of the fine-wire electrodes were attached to preamplifiers on the forearm and hand (Fig. 2).

In each volunteer and during each task, the activity of eight thumb muscles was recorded simultaneously; four intrinsic thumb muscles: $\mathrm{m}$. opponens pollicis (OP), $\mathrm{m}$. flexor pollicis brevis (FPB), $\mathrm{m}$. abductor pollicis brevis (APB) and the m. adductor pollicis (ADP), and four extrinsic thumb muscles: $\mathrm{m}$. extensor pollicis longus (EPL), $\mathrm{m}$. extensor pollicis brevis (EPB), m. abductor pollicis longus (APL), and $\mathrm{m}$. flexor pollicis longus (FPL). These muscles were selected based on their reported activity during specific thumb movements (Kaufman et al., 1999).
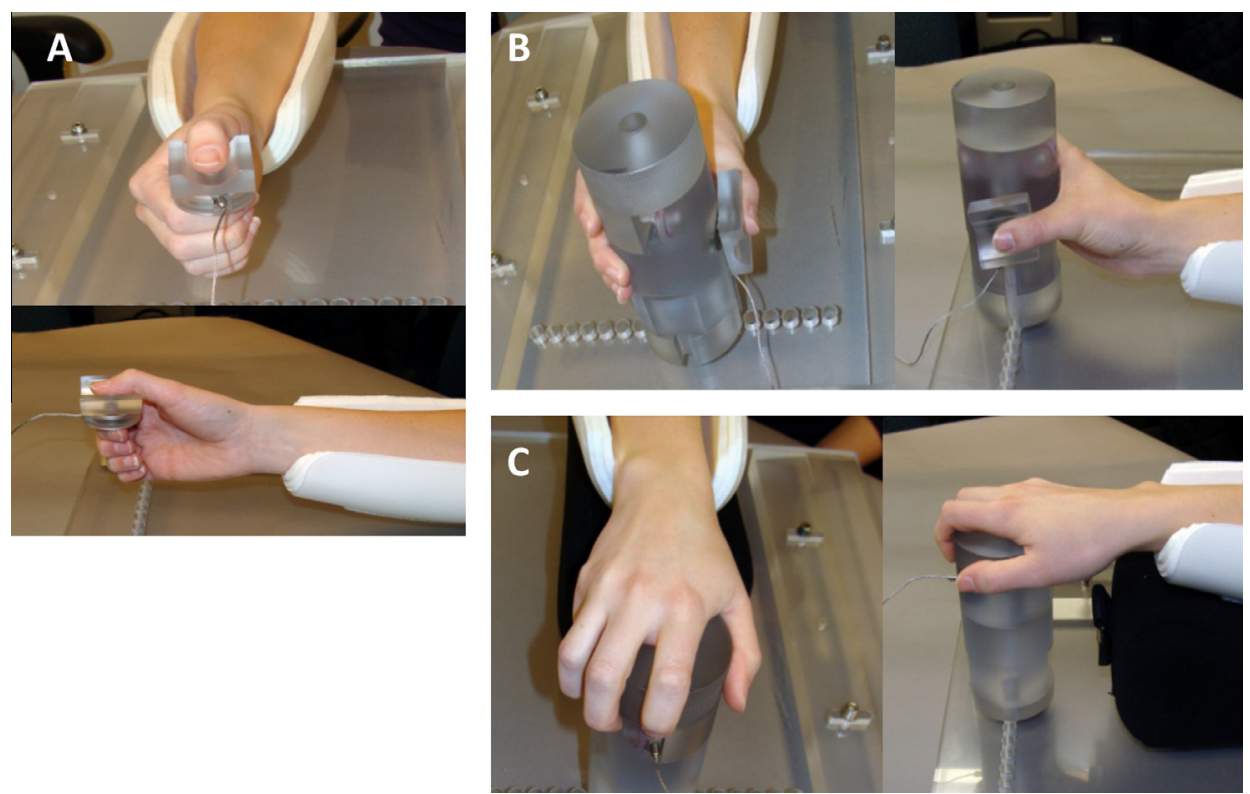

Fig. 1. Hand positioning in the polycarbonate jig during the three functional tasks. A: lateral key pinch, B: power grasp and C: jar twist. 


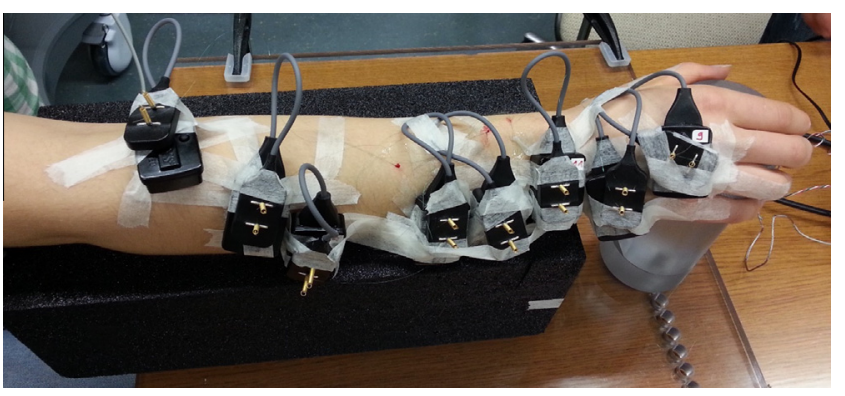

Fig. 2. Electrode insertion and pre-amplifier placement of 8 electrodes on the forearm of a volunteer.

\subsection{EMG analysis}

Raw EMG signals were imported into EMGworks 4.0 Analysis software (Delsys, Inc., Boston, USA) for processing. The EMG data were high-pass, 2nd order Butterworth filtered at $10 \mathrm{~Hz}$. The amplitude of the EMG signal was obtained by deriving the rootmean square of the signal over a $0.125 \mathrm{~s}$ moving window, resulting in full-wave rectification and smoothing of the raw signal. Muscle activity of the opponens pollicis muscle was omitted from the analyses due to uncertainty about the precision of electrode placement and subsequent signal quality. Only recordings of submaximal tasks were used in our analysis as these best represent functional activities of daily life. EMG data of one subject was excluded from the analysis due to missing data (only 4 of the 8 muscles were correctly sampled).

The absolute EMG values (in Volts) were used to explore subject-specific muscle activation patterns during the three different tasks. Per subject, the mean absolute EMG amplitude was calculated for each muscle during the submaximal tasks. To detect task-specific muscle activation patterns across all subjects, the absolute EMG data were scaled to percentages of maximal activation. For each subject and for each muscle, the highest EMG signal amplitude obtained during the nine recordings (three per task) was used as a scaling value. Subsequently, the EMG data were expressed as a percentage of this peak amplitude. By averaging the mean percentages of all subjects for every muscle, it is possible to detect if specific muscles on average increase or decrease their activity when switching between tasks.

\subsection{Statistical analysis}

A Shapiro-Wilk test was used to evaluate the normality of the force data ( $\mathrm{W}=0.09456, \mathrm{p}=0.59$ ). To determine the influence of electrode placement on force production, a Wilcoxon signed-rank test was performed on the force data recorded before and after implantation of the fine-wire electrodes.

To compare the scaled mean EMG values between the intrinsic thumb muscles (APB, ADP, FPB) and the extrinsic thumb muscles (EPB, EPL, APL, FPL), a Mann-Whitney test was performed on the scaled EMG values for each task. The differences in EMG activity between tasks was explored by comparing the scaled EMG activities for each individual muscle with a repeated measure ANOVA. Overall effects of task, subject, muscle and their interaction effects were tested with a three-way ANOVA. All ANOVAs were corrected using a Sidak's post hoc test.

Graphpad Prism (GraphPad Software Inc., La Jolla, USA) and SAS (SAS Institute Inc., Cary, USA) were used for statistical testing. Significance level was set at $\mathrm{p}<0.05$.

\section{Results}

3.1. Muscle activity patterns during lateral key pinch, power grasp and jar twist

Muscle activity patterns for each subject and each task are shown in Fig. 3A-C. The radar plots illustrate the inter-individual variability in muscle recruitment across tasks. The three-way ANOVA showed that scaled EMG values are significantly different between subjects $(\mathrm{F}=18.67, \mathrm{df}=9, \mathrm{p}<0.001)$, that scaled EMG activity varies significantly between muscles $(F=184,01, \mathrm{df}=6$, $\mathrm{p}<0.001$ ) and that the overall activation pattern of the thumb muscles remains largely unchanged between tasks $(F=0.02$, $\mathrm{df}=2, \mathrm{p}=0.98$ ). The interaction effects between task, muscle and subject all explain substantial parts of the variation in EMG activity; task $*$ muscle $(\mathrm{F}=2.92, \quad \mathrm{df}=12, \quad \mathrm{p}=0.002), \quad$ task $*$ subject $(\mathrm{F}=1.95, \quad \mathrm{df}=18, \quad \mathrm{p}=0.019) \quad$ and muscle $*$ subject $\quad(\mathrm{F}=39.78$, $\mathrm{df}=54, \mathrm{p}<0.001)$ are all significant.

The scaled EMG activity of each muscle across tasks is depicted in Fig. 4. The repeated measures ANOVA showed that most muscles show no change in activity when switching between tasks. For example, APL is consistently activated at $95 \%$ of its maximal activity during lateral key pinch, power grasp or jar twist $(F=0.8743$, $\mathrm{df}=2, \mathrm{p}=0.40$ ). The only muscle where we found statistically significant differences in muscle activation between tasks is the ADP $(\mathrm{F}=4.074, \mathrm{df}=2, \mathrm{p}=0.04)$, demonstrating a significant decrease in activation when shifting from power grasp to jar twist (after Sidak's post hoc correction; $\mathrm{p}=0.03$ ).

\subsection{Intrinsic and extrinsic thumb muscle activity}

The scaled muscle activity of the intrinsic and extrinsic thumb muscles for each task are presented in Fig. 5. We found significant differences between intrinsic and extrinsic muscle activity during all tasks. The activity of the extrinsic muscles was significantly higher than that of the intrinsic muscles during lateral key pinch (Mann-Whitney $U=241, n_{1}=30 n_{2}=40, P<0.001$ two-tailed), power grasp (Mann-Whitney $U=192, n_{1}=30 n_{2}=40, P<0.001$ two-tailed) and jar twist (Mann-Whitney $U=189, n_{1}=30 n_{2}=40$, $P<0.001$ two-tailed). Yet, inter-individual variation is substantial, with standard deviations from the mean of over $20 \%$.

\subsection{Influence of electrode insertion on force production}

The effect of electrode placement on force production is shown in Fig. 6. Force production was significantly decreased after electrode insertion in every task (lateral key pinch: $t=7.133, \mathrm{df}=1$, $\mathrm{p}<0.001$; power grasp: $\mathrm{t}=3.906, \mathrm{df}=10, \mathrm{p}<0.001$, jar twist: Wilcoxon $\mathrm{W}=-66, \mathrm{p}=0.001)$. The mean force reduction amounted to $17.0 \%(13.72 \mathrm{~N})$ during lateral key pinch, to $11.3 \%(9.91 \mathrm{~N})$ for power grasp and to $17.9 \%(18.45 \mathrm{~N})$ for jar twist.

\section{Discussion}

Contrary to our expectations, we did not find a task-specific activation of the thenar muscles. Instead, a subject-specific activation pattern was observed in which each subject used a consistent muscle activation pattern across tasks, with large differences between subjects. We found a strong activation of all tested thenar muscles in each of the tasks, with no difference in muscle activation between lateral key pinch, power grasp or jar twist tasks (at $80 \%$ effort) except for the adductor pollicis muscle. Grouped extrinsic muscle activity was higher than intrinsic muscle activity in all tasks. 
lateral key pinch

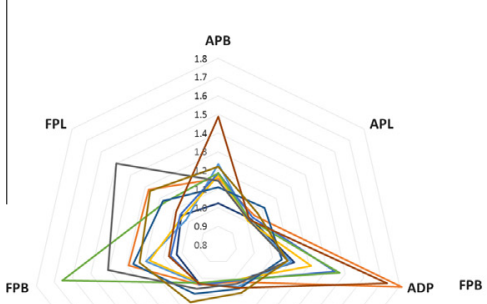

EPL

A power grasp

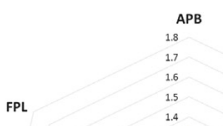

FPL

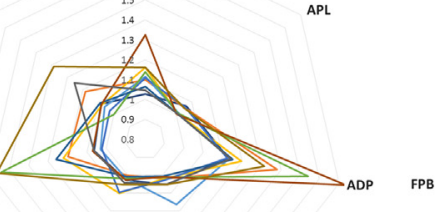

jar twist

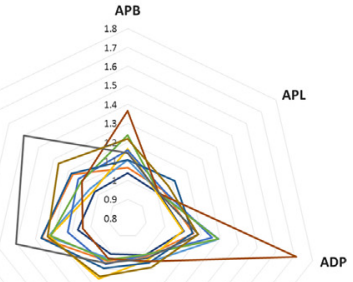

EPL

EPB

C

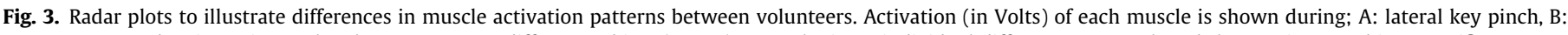

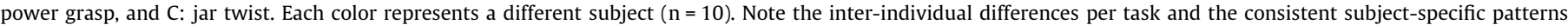

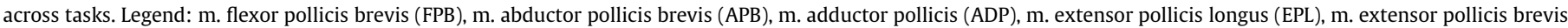

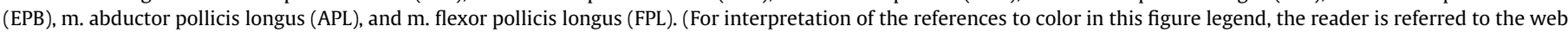
version of this article.)

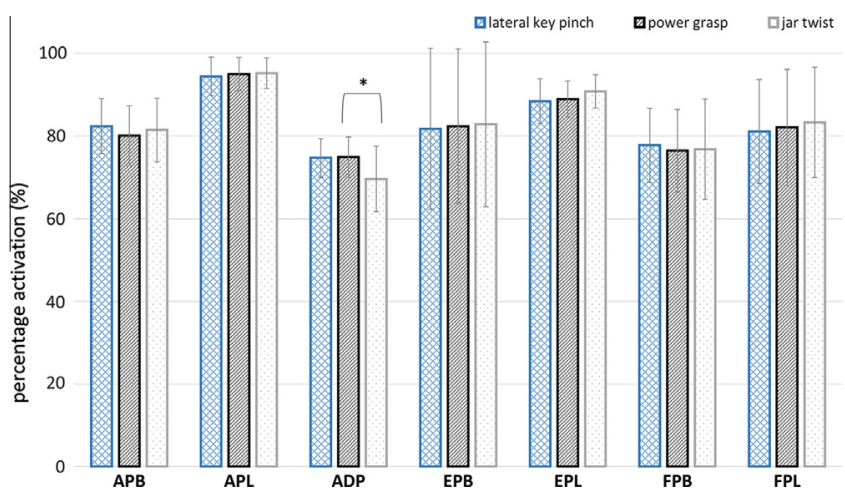

Fig. 4. Mean and standard deviation of scaled EMG activity (\%) for each muscle across tasks. Legend: lateral key pinch=blue diamonds, power grasp=black stripes, jar twist = grey dots. Asterisk indicates significant differences between tasks. (For interpretation of the references to color in this figure legend, the reader is referred to the web version of this article.)

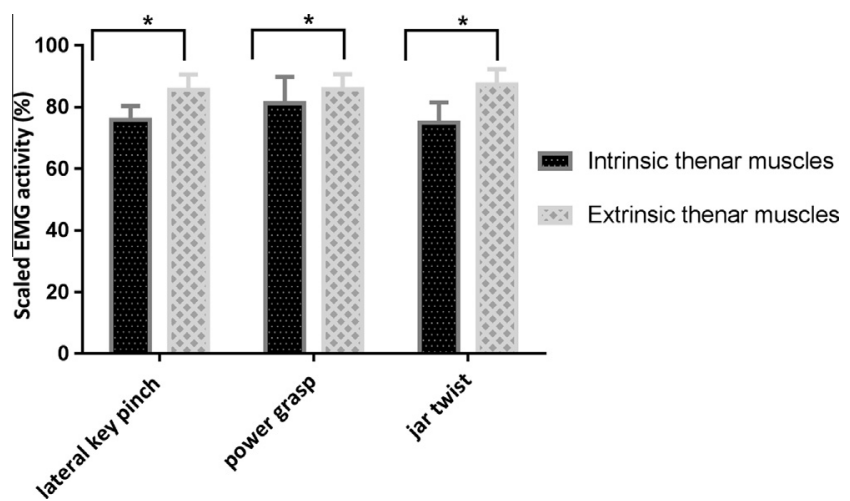

Fig. 5. Mean and standard deviation of scaled EMG activity (\%) of the intrinsic and extrinsic thumb muscles for each task at $80 \%$ of maximal voluntary contraction. Asterisk indicates significant differences between intrinsic and extrinsic muscle activity.

\subsection{Subject-specific muscle activation patterns}

Inter-individual variability in hand EMG activity has been reported by other studies. Kilbreath and Gandevia found that when

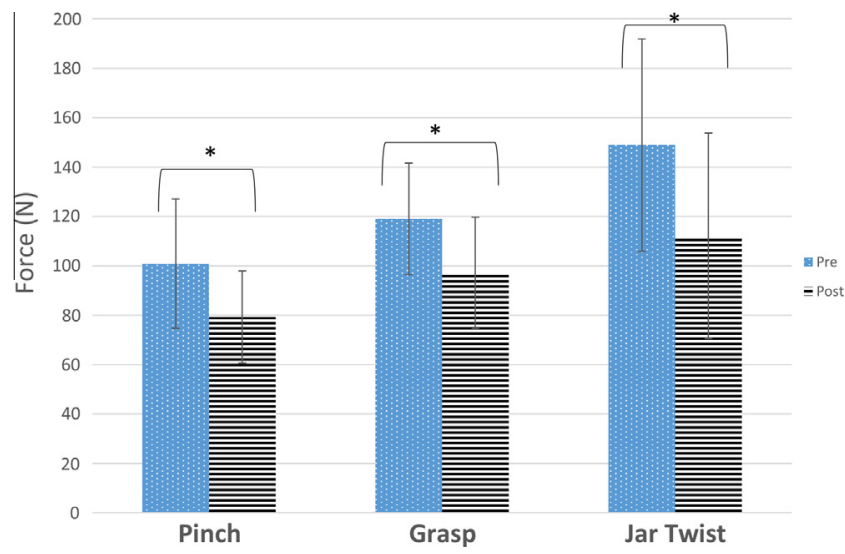

Fig. 6. Mean and standard deviation of maximum force production pre- and postelectrode placement. Legend: blue $=$ pre, orange $=$ post, Asterisk marks significant differences between both conditions. (For interpretation of the references to color in this figure legend, the reader is referred to the web version of this article.)

grasping cylinders using a pinch grip, the scaled EMG activity of the flexor digitorum profundus, first dorsal interosseous, adductor pollicis and flexor pollicis longus muscles varied significantly between subjects. The authors suggest that strategies used to hold the object must vary widely between subjects (Kilbreath and Gandevia, 1994).

A study by Johanson and colleagues also support the concept of individual motor strategies for pinch tasks. They found interindividual differences in EMG activity during key pinch and opposition pinch tasks at 50\% MVC in the extensor pollicis longus, extensor pollicis brevis, abductor pollicis brevis and flexor pollicis brevis muscles. Inter-individual variability was limited in the flexor pollicis longus, opponens pollicis, adductor pollicis and first dorsal interosseous muscles (Johanson et al., 1996). Although the forces in the current study were higher (80\% effort) and the tasks different, a similar pattern can be seen with high inter-individual variability of extensor pollicis brevis and less variability of the adductor pollicis muscle.

\subsection{Task-specific muscle activation patterns}

Despite the variability in activation of the adductor pollicis muscle across subjects, we did discern a significant decrease in 
muscle activation when switching from power grip to a jar twist task. Overall, we did observe some small differences between tasks but no overarching task-specific recruitment of the thenar muscles. The absence of a task-specific muscle recruitment could be due to the fact that all three tasks were forceful activities at $80 \%$ of their maximal performance. Possibly, a high recruitment of both the intrinsic and extrinsic thenar muscles is required in such forceful tasks to stabilize the joints, contrary to the situation in the low force range where intrinsic muscles act as precision force regulators (Maier and Hepp-Reymond, 1995). Also, lateral key pinch, power grasp and jar twist represent a multi-joint and multimuscular system with a certain muscle redundancy. This redundancy might explain the co-activation of all tested thenar muscles and the large inter-individual variation in muscle activation patterns observed in all tasks. Importantly, this suggests that many different activation patterns can lead to the same task performance. This has also previously been reported for a precision grip task in a study by Maier and Hepp-Reymond (1995) and in the before mentioned research by Kilbreath and Gandevia in which they demonstrated that when the grip was changed from a pinching to an encircling grip, while grasping and lifting different cylinders, activity of all four tested muscles altered significantly. This in contrast to the current study, where we found no differences in activity between tasks. These opposite finding could be related to the weight of the grasped cylinder. The cylinders their subjects needed to grasp and lift, varied between 0.25 and $0.5 \mathrm{~kg}$. Representing no more than $50 \%$ of maximum effort. The fact that the range of their collected EMG values increased when muscle activity increased, underlines the potential influence of effort (Kilbreath and Gandevia, 1994).

Johanson and colleagues did also find differences in activation patterns when switching between tasks, but they focused on key and opposition pinch in stable and unstable tasks (Johanson et al., 2001). During functional pinch, they found a strong activation of the abductor pollicis and extensor pollicis longus muscles. Such specific high activation of only the abductor pollicis longus during lateral key pinch was not found in our study, even though comparison is difficult due to the different experimental setup and protocol.

\subsection{Study limitations}

Although our sample is larger than that of most previous studies (e.g., $n=7$ : (Birdwell et al., 2013; Johanson et al., 2001; ValeroCuevas et al., 2003); $n=6$ : (Maier and Hepp-Reymond, 1995)), a sample size of ten subjects remains relatively low. Sampling a larger cohort is, however, difficult seen the invasiveness of the protocol, with insertion of eight electrodes in the forearm and hand of volunteers while performing isometric tasks.

In our protocol, based on the recent publication of Perotto (1994), we used a palmar approach to insert the electrodes into the intrinsic thumb muscles. However, piercing the thenar clearly had an influence on force production during the experiments. A dorsal approach for insertion would probably have been less uncomfortable for the participants. This has also been suggested in earlier publications of Valero-Cuevas and colleagues (Burgar et al., 1997; Valero-Cuevas et al., 1998).

All tasks were executed at $80 \%$ of their maximal performance, which might obscure differences in muscle activation patterns between tasks due to the high need for joint stabilization. Sampling EMG-data during a stepwise increase in force production (e.g., 10\%, 20\%, 30\%) could give a better appraisal of the differential recruitment of intrinsic and extrinsic thenar muscles.

Finally, we sampled the activity of eight thenar muscles simultaneously during the three isometric tasks, but had to exclude the recordings of the opponens pollicis muscle from our analysis. Also the activity of the first dorsal interosseous muscle was not recorded during the tasks, although this muscle might play an important role in the stabilization of the thumb (Johanson et al., 2001; Kozin et al., 1999; Maier and Hepp-Reymond, 1995).

\subsection{Clinical relevance}

In our study, we focused on three functional tasks corresponding to activities of daily living. The ability to execute these tasks is important for the independence of a person. In people with advanced $\mathrm{OA}$ at the thumb base, these manipulative skills are often compromised due to joint pain or reduced joint mobility (Wilder et al., 2006). Compressive forces, joint laxity and muscle imbalance may contribute to the development of the disease but, to date, the precise pathomechanism remains unclear. OA of the TMC joint might develop in a way comparable to medial compartment OA of a varus knee (Edmunds, 2011). In both cases, OA starts at the area where the highest compressive forces occur. However, in contrast to the knee which is a weight-bearing joint, loading of the TMC joint mainly results from contraction of the intrinsic and extrinsic thumb muscles. Therefore, muscular loading on the TMC joint could play an important role in the development of TMC OA. To get more accurate estimations of joint loading, a more complex joint model is needed which takes the different activation levels of the thumb muscles into account. The data collected in the present study can be used as input for an EMG-driven model or help validate existing musculoskeletal models. Expanding on this concept, it would be highly interesting to compare thumb muscle activation patterns of OA patients with the EMG data of healthy controls from the current study. However, such data might not be easy to acquire and the substantial inter-individual variability found in our study might complicate a meaningful comparison.

\section{Conclusion}

The thumb muscles display a high variability in activity during functional tasks of daily life. The results of this study suggest that to produce a substantial amount of force, a well-integrated, but subject-specific, co-contraction between the intrinsic and extrinsic thumb muscles is necessary. Information on muscle activation patterns during functional tasks is important to achieve a better understanding of the development of TMC OA and for establishing or validating an accurate musculoskeletal model of the thumb.

\section{Conflict of interest}

This research was partially funded by the Materialise-Kulak Chair on Hand Surgery.

\section{Acknowledgements}

We are grateful to the volunteers who participated in this study and to Dr. Hans Pottel for statistical advice.

\section{References}

Birdwell, J.A., Hargrove, L.J., Kuiken, T.A., Weir, R.F., 2013. Activation of individual extrinsic thumb muscles and compartments of extrinsic finger muscles. J. Neurophysiol. 110, 1385-1392.

Burgar, C.G., Valero-Cuevas, F.J., Hentz, V.R., 1997. Fine-wire electromyographic recording during force generation: application to index finger kinesiologic studies. Am. J. Phys. Med. Rehabilit./Assoc. Acad. Physiatrists 76, 494-501.

Cooney, W.P., An, K.N., Daube, J.R., Askew, L.J., 1985. Electromyographic analysis of the thumb: a study of isometric forces in pinch and grasp. J. Hand Surg. 10, $202-$ 210.

Cooney, W.P., Chao, E.Y., 1977. Biomechanical analysis of static forces in the thumb during hand function. J. Bone Joint Surg. Am. 59, 27-36. 
Corneil, B.D., Goonetilleke, S.C., Peel, T.R., Green, K.A., Welch, I.D., 2012. Ultrasoundguided insertion of intramuscular electrodes into suboccipital muscles in the non-human primate. J. Electromyogr. Kinesiol. 22, 553-559.

Crisco, J.J., Halilaj, E., Moore, D.C., Patel, T., Weiss, A.P.C., Ladd, A.L., 2015. In vivo kinematics of the trapeziometacarpal joint during thumb extension-flexion and abduction-adduction. J. Hand Surg. 40, 289-296.

Eaton, R., Glickel, S., Barron, A., 2000. Basal joint arthritis of the thumb. J. Am. Acad. Orthop. Surg. 8, 314-323.

Edmunds, J.O., 2011. Current concepts of the anatomy of the thumb trapeziometacarpal joint. J. Hand Surg. 36, 170-182.

Halilaj, E., Moore, D.C., Laidlaw, D.H., Got, C.J., Weiss, A.P.C., Ladd, A.L., Crisco, J.J., 2014. The morphology of the thumb carpometacarpal joint does not differ between men and women, but changes with aging and early osteoarthritis. J. Biomech. 47, 2709-2714.

Halilaj, E., Rainbow, M.J., Got, C.J., Moore, D.C., Crisco, J.J., 2013. A thumb carpometacarpal joint coordinate system based on articular surface geometry. J. Biomech. 46, 1031-1034.

Johanson, M.E., Skinner, S.R., Lamoreux, L.W., 1996. Phasic relationships of the intrinsic and extrinsic thumb musculature. Clin. Orthop. Relat. Res. 322, 120130.

Johanson, M.E., Valero-Cuevas, F.J., Hentz, V.R., 2001. Activation patterns of the thumb muscles during stable and unstable pinch tasks. J. Hand Surg. 26, 698705.

Kaufman, K.R., An, K.N., Litchy, W.J., Cooney, W.P., Chao, E.Y.S., 1999. In-vivo function of the thumb muscles. Clin. Biomech. 14, 141-150.

Kilbreath, S.L., Gandevia, S.C., 1994. Limited independent flexion of the thumb and fingers in human subjects. J. Physiol. 479, 487-497.

Konrad, P., 2005. The abc of emg. In: A Practical Introduction to Kinesiological Electromyography, first ed. Noraxon, Scottsdale.

Kozin, S.H., Porter, S., Clark, P., Thoder, J.J., 1999. The contribution of the intrinsic muscles to grip and pinch strength. J. Hand Surg. 24, 64-72.

Maier, M.a., Hepp-Reymond, M.C., 1995. EMG activation patterns during force production in precision grip. II. Muscular synergies in the spatial and temporal domain. Exp. Brain Res. Experimentelle Hirnforschung. Experimentation cerebrale $103,123-136$.

Perotto, A., 1994. Anatomical Guide for the Electromyographer: The Limbs and Trunk, fifth ed. Charles C Thomas Publisher, Illinois.

Rudroff, T., Staudenmann, D., Enoka, R.M., 2008. Electromyographic measures of muscle activation and changes in muscle architecture of human elbow flexors during fatiguing contractions. J. Appl. Physiol. (Bethesda, Md.: 1985) 104, 1720 1726.

Solomonow, M., Baratta, R., Bernardi, M., Zhou, B., Lu, Y., Zhu, M., Acierno, S., 1994. Surface and wire EMG crosstalk in neighbouring muscles. J. Electromyogr. Kinesiol. 4 (3), 131-142.

Türker, K.S., 1993. Electromyography: some methodological problems and issues. J. Am. Phys. Ther. Assoc., 659-660

Valero-Cuevas, F.J., Johanson, M.E., Towles, J.D., 2003. Towards a realistic biomechanical model of the thumb: the choice of kinematic description may be more critical than the solution method or the variability/uncertainty of musculoskeletal parameters. J. Biomech. 36, 1019-1030.

Valero-Cuevas, F.J., Zajac, F.E., Burgar, C.G., 1998. Large index-fingertip forces are produced by subject-independent patterns of muscle excitation. J. Biomech. 31, 693-703.

Wilder, F.V., Barrett, J.P., Farina, E.J., 2006. Joint-specific prevalence of osteoarthritis of the hand. Osteoarthritis Cartilage 14, 953-957.

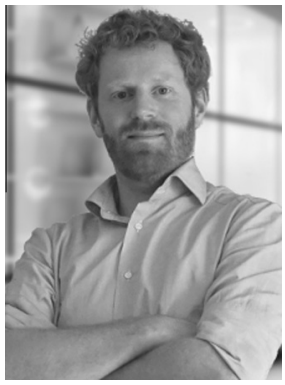

Faes D. Kerkhof obtained a bachelor degree within the domain of physical education at the University of Applied Sciences in Eindhoven. He worked as a climbing and skiing instructor in Austria and France, before receiving his MSc in Human Movement Sciences from the University of Groningen in the Netherlands. He moved to Belgium where he currently combines his passion for teaching and science as an assistant lecturer and PhD student at the Faculty of Medicine in the University of Leuven. Current research topics are; determining thumb kinematics using dynamic CT, intrinsic thumb muscle activity during activities of daily life and obtaining anatomical information via medical imaging, opto-electronic and magnetic digitization.

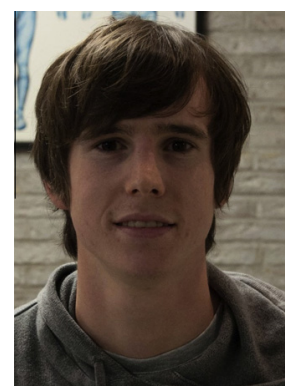

Gertjan Deleu, after acquiring a MSc in physiotherapy, Gertjan Deleu works as a physiotherapist and is one of the revalidation trainers for soccer team KV Kortrijk.

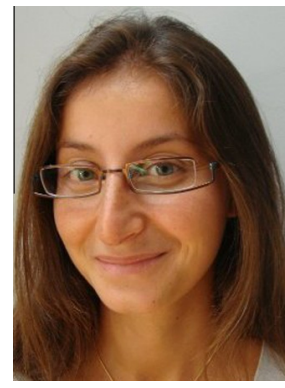

Priscilla D'Agostino is a qualified orthopaedic surgeon specialised in hand and wrist surgery, peripheral nerve surgery, arthroscopic surgery and microsurgery. She is licensed by the Belgian Ministry of Public Health since 2009 and has been trained alongside internationally renowned specialists in centres of excellence in hand surgery. Priscilla's clinical work is exclusively dedicated to upper limb surgery and in particular all conditions of the hand, wrist, forearm and elbow. She is an active member of several renowned European and international societies related to her field of expertise. In addition, Priscilla is involved in several scientific research projects regarding the upper limb and is currently working on a $\mathrm{PhD}$ project at the University of Leuven which specifically focusses on the anatomy and biomechanics of the trapeziometacarpal joint.

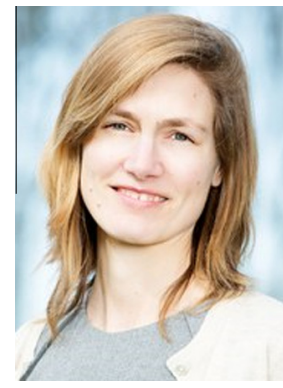

Evie Vereecke is professor of Anatomy and head of the Jan Palfijn Anatomy Lab. She obtained a PhD in 2006 at the Department of Biology from the University of Antwerp (Belgium) with a thesis on the bipedal locomotion of apes (gibbon and bonobo). Evie continued her research activities in the domain of functional anatomy and primate locomotion at the University of Liverpool (UK), where she spent three years as a lecturer in anatomy. She moved back to Belgium in 2008 to take up a position as senior research policy advisor at the Research Foundation Flanders, but after two years she moved back to academia. Evie Vereecke obtained a tenure-track position at the Faculty of Medicine in the University of Leuven in 2010 and started a research group focusing on the function and evolution of the human musculoskeletal system. 